TOKUSHIMA 95-03

(hep-ph/9505249)

May 1995

\title{
Indications on the Higgs-Boson Mass from the LEP Data
}

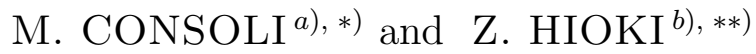 \\ a) Istituto Nazionale di Fisica Nucleare - Sezione di Catania \\ Corso Italia, 57 - I 95129 Catania - ITALY \\ b) Institute of Theoretical Physics, University of Tokushima \\ Tokushima 770 - JAPAN
}

\begin{abstract}
We update our previous analysis on the Higgs mass $m_{h}$ and the QCD coupling $\alpha_{s}\left(=\alpha_{s}\left(M_{z}\right)\right)$ by using the LEP data after the 1995 Winter Conferences. For $m_{t}=180 \mathrm{GeV}$ we find evidence for a rather large value of the Higgs mass in the range 500-1000 GeV, in agreement with the indications from the $\mathrm{W}$ mass.
\end{abstract}

\footnotetext{
${ }^{*}$ E-mail address: consoli@vxcern.cern.ch

**)E-mail address: hioki@ias.tokushima-u.ac.jp
} 
Strong evidence for the top quark has been observed by CDF and D0 Collaborations independently [目]. We have now only one yet-undiscovered particle left in the framework of the standard electroweak model. A lot of experimental and theoretical efforts should be made toward this particle, i.e., the Higgs boson. It is not that easy to draw its indirect information from existing experimental data since the Higgs mass $m_{h}$ enters the one-loop electroweak predictions only logarithmically. Therefore, at present, one can only hope to separate out the heavy Higgs-mass range (say $m_{h} \sim 500-1000 \mathrm{GeV}$ ) from the low mass regime $m_{h} \sim 100$ $\mathrm{GeV}$ as predicted, for instance, from supersymmetric theories. Such analyses are, however, still very important and indispensable for future experiments at, e.g., LHC/NLC.

In our previous work [2], we have performed a detailed comparison of the LEP data presented at the 1994 Glasgow Conference [3] with the standard model for the various observables. There we obtained some interesting information on $m_{h}$ and the strong-interaction coupling constant at the Z-mass scale $\alpha_{s}=\alpha_{s}\left(M_{z}\right)$. In this note, we shall update this analysis by using the more precise data from the 1995 Winter Conferences as reported by ALEPH, DELPHI, L3 and OPAL in [4] and the new top mass $m_{t}=180 \pm 12 \mathrm{GeV}$ [1].

For our analysis, we used in [2] the disaggregated data, just as presented by the experimental Collaborations, without attempting any average of the various results. This type of analysis is interesting by itself to point out the indications of the various sets of data since even a single measurement, if sufficiently precise, can provide precious information. At the same time, since the LEP data are becoming so precise, before attempting any averaging procedure one should first analyze the various measurements with their errors and check that the distribution of the results fulfills the requirements of gaussian statistics. Without this preliminary analysis one may include uncontrolled systematic effects which can sizeably affect the global averages.

For instance, in [5] a detailed analysis of the relative magnitude of the hadronic 
and leptonic widths for the different channels of the various experiments was performed. Starting from the LEP data presented at the Glasgow Conference [3] and using a Monte Carlo method to generate a large number of "a priori" equivalent copies, one finds [5] that the probability of the original LEP population is extremely small $\left(3.8 \times 10^{-4}\right)$. Therefore, the meaning of the global average $R=\Gamma_{h} / \Gamma_{l}=20.795 \pm 0.040$ presented in [3] is unclear and substantial systematic effects have to be invoked to understand the distribution of the various measurements.

In the following, we develop analyses similar way to our previous work [2] in order to make the comparison with it easy and convenient. We shall first restrict to a fixed value of the top-quark mass $m_{t}=180 \mathrm{GeV}$ and discuss the indications for the Higgs mass. The experimental data relevant for our analysis are presented in Table I. These are the available, individual results from the various Collaborations as quoted in [4] and the meaning of the various quantities is the same as in [4]. The theoretical predictions in Table II, for several values of $\alpha_{s}$ and $m_{h}$ representative of the overall situation, have been obtained with the computer code TOPAZ0 by Montagna et al. [6]. Finally, in Tables III-VI we report the partial and total $\chi^{2}$ for the various experiments and in Table VII the sum of the $\chi^{2}$ for the four Collaborations.

Tables I - VII

We find here again some tendencies in the data: The global values of the $\chi^{2}$ in Table VII confirm that $\alpha_{s}$ lies at $\sim 3 \sigma$ from the DIS prediction $\alpha_{s}=0.113 \pm 0.005$ (here, our result is in very good agreement with the general analysis of [7] which gives $\left.\alpha_{s}=0.127 \pm 0.005\right)$. Further, by inspection of Table I one finds evidences for some systematic effect in the $\tau$ F-B asymmetry. This effect seems to be common to all experiments and it is confirmed by the following remark. Let us consider 
the global averages reported in [4]

$$
\begin{aligned}
& A_{F B}^{o}(e)=0.0154 \pm 0.0030 \\
& A_{F B}^{o}(\mu)=0.0160 \pm 0.0017 \\
& A_{F B}^{o}(\tau)=0.0209 \pm 0.0024
\end{aligned}
$$

and transform the averages for $A_{e}$ and $A_{\tau}$, 4

$$
A_{e}=0.137 \pm 0.009, \quad A_{\tau}=0.140 \pm 0.008
$$

into "equivalent" F-B asymmetries by using the standard model formula

$$
A_{F B}^{o}(1)=\frac{3}{4} A_{e}^{2}, \quad A_{F B}^{o}(2)=\frac{3}{4} A_{e} A_{\tau}
$$

We find

$$
A_{F B}^{o}(1)=0.0141 \pm 0.0019, \quad A_{F B}^{o}(2)=0.0144 \pm 0.0018
$$

in very good agreement with Eqs.(1,2) but not with Eq.(3). Therefore, there may be some problem in the direct measurement of $A_{F B}^{o}(\tau)$ since all other measurements are in excellent agreement with each other. Just to have an idea of the effect, if the data for the $\tau$ F-B asymmetry are omitted in the evaluation of the $\chi^{2}$ we find the results illustrated in Table VIII which one should compare with Tables III- VII. The "bulk" of the LEP data, namely those well consistent with each other, show no preference for a light Higgs boson and the best values of the $\chi^{2}$ are obtained for a large value of $m_{h}$, just as in the case of the W mass reported in 8 . 17

\section{Table VIII}

\footnotetext{
${ }^{\sharp 1}$ The latest world average of the $\mathrm{W}$-mass is $M_{w}=80.27 \pm 0.14 \mathrm{GeV}$. Comparing it with the one computed from $M_{z}$, we find not only that the central value of $m_{h}$ must be more than 1 $\mathrm{TeV}$ but also that $m_{h}=100 \mathrm{GeV}$ is disfavored (though at $1 \sigma$ level). See [9] for more details.
} 
Finally, to have an idea of the dependence on $m_{t}$, we report in Table IX and Table $\mathrm{X}$ the total $\chi^{2}$ for $m_{t}=170,180$ and $190 \mathrm{GeV}$ including all data or excluding $A_{F B}^{o}(\tau)$. As first noticed by Ellis et al. [10, 11, by increasing (decreasing) the top-quark mass a larger (smaller) value of $m_{h}$ is favoured and the shape of the $\chi^{2}$ is well consistent with all values of the Higgs mass. For $m_{t}=180 \mathrm{GeV}$, however, Table IX and Table $\mathrm{X}$ give rather different information and it becomes crucial to include the more problematic data for $A_{F B}^{o}(\tau)$ to accommodate values $m_{h} \sim 100$ $\mathrm{GeV}$.

\section{Tables IX and X}

We have of course no mind to say that Tables VIII and X represent a more faithful representation of the real physical situation than Tables VII and IX. Most likely, our results suggest only that further improvement in the data taking is needed for a definitive answer. We may, however, conclude that it is dangerous to focus on a light-mass region in Higgs searches at future experiments. Also, our analysis, confirming the conclusions of [2], shows that the possibility to obtain precious information on the Higgs mass is not unrealistic when the top-quark mass will be measured with a higher precision at the Tevatron.

To better appreciate this point, let us consider the hypothetical situation where $m_{t}$ would be known to be $180 \mathrm{GeV}$ to very high accuracy (at the end of the century the combined $\mathrm{CDF}+\mathrm{D} 0$ determination should provide an overall error $\Delta m_{t}= \pm 3 \mathrm{GeV}[12]$ ). In this case, what would we deduce from the present LEP results? On one hand, we find a clear signal for a heavy Higgs from the very precise data of the OPAL Collaboration (see Table VI) which completely confirms the indications from the $\mathrm{W}$ mass. In fact, by inspection of Table VI, the two pairs corresponding to $m_{h}=100 \mathrm{GeV}$ lie outside the $95 \%$ C.L. contour $\left(\Delta \chi^{2}=+6.1\right)$ in the two-parameter space $\left(\alpha_{s}, m_{h}\right)$. This effect is independent on $\alpha_{s}$ since the pair $(0.13,100)$ has a total $\chi^{2}=13.87$ with a difference $\Delta \chi^{2}=+7$ 
with respect to the configuration $(0.13,1000)$ shown in Table VI. On the other hand, in this hypothetical scenario for the top mass, the OPAL trend is not confirmed by the other Collaborations since, by subtracting out the OPAL data from Table VII, ALEPH+DELPHI+L3 give no particular indications. Indeed, their total $\chi^{2}=27.9,29.1,28.6$ and 31.2 , for the four pairs $\left(\alpha_{s}, m_{h}\right)$ considered in our analysis, produce a maximum difference $\Delta \chi^{2}=+3.3$ so that even if the top mass would be known with infinite precision to be $180 \mathrm{GeV}$ their data would be well consistent with all values of $m_{h}$ and $\alpha_{s}$.

Therefore, if we really want to explore the full potentiality of LEP for a precise determination of $m_{h}$ (and $\alpha_{s}$ ) in the standard electroweak theory, much more stringent tests have to be performed. As stressed in [2], a precise scanning of the $\mathrm{Z}$ resonance with 4 or more points at high statistics off peak cannot be postponed anymore ( $\sim 90 \%$ of the total events have been collected at the pole ). Further, a high luminosity phase of LEP I, where each Collaboration will detect millions of Z's per run and the purely statistical errors will become negligible, is needed to obtain a definitive consistency check of the systematics of the various experiments.

\section{ACKNOWLEDGEMENTS}

We thank Giampiero Passarino for many useful discussions.

\section{References}

[1] CDF Collaboration : F. Abe et al., Phys. Rev. Lett. 74 (1995), 2626;

D0 Collaboration : S. Abachi et al., Phys. Rev. Lett. 74 (1995), 2632.

[2] M. Consoli and Z. Hioki, Mod. Phys. Lett. A10 (1995), 845. 
[3] The LEP Collaborations, A Combined preliminary data on $Z$ parameters from the LEP experiments and constraints on the Standard Model, preprint CERN/PPE/94-187, 1994.

[4] The LEP Collaborations, A Combination of preliminary LEP Electroweak Results for the 1995 Winter Conferences, preprint LEPEWWG/95-01, ALEPH 95-038, DELPHI 95-37, L3 Note 1736, OPAL Note TN284, 17 March 1995.

[5] M. Consoli and F. Ferroni, Phys. Lett. B349 (1995), 375.

[6] G. Montagna, O. Nicrosini, G. Passarino, F. Piccinini and R. Pittau, Comput. Phys. Commun. 76 (1993), 328.

[7] P. Langacker, Tests of the Standard Model and Searches for New Physics, to be published in Precisions Tests of the Standard Electroweak Model, World Scientific, Singapore 1994 (hep-ph/9412361).

[8] Z. Hioki and R. Najima, Mod. Phys. Lett. A10 (1995), 121.

[9] Z. Hioki, preprint TUM-T31-80/94 (hep-ph/9410288) (to appear in Int. J. Mod. Phys. A).

[10] J. Ellis, G. L. Fogli and E. Lisi, Phys. Lett. B333 (1994) 118.

[11] J. Ellis, G. L. Fogli and E. Lisi, Phys. Lett. B318 (1993) 148 and older references quoted therein.

[12] M. Mangano, Talk given at the '95 LEP Workshop, 10-12 April, Genova. 


\section{TABLES}

\begin{tabular}{lcccc} 
& ALEPH & DELPHI & L3 & OPAL \\
\hline \hline$\Gamma_{z}(\mathrm{MeV})$ & $2493.2 \pm 5.8$ & $2494.1 \pm 5.5$ & $2504.0 \pm 5.3$ & $2496.0 \pm 5.2$ \\
\hline$\sigma_{\text {had }}(\mathrm{nb})$ & $41.62 \pm 0.10$ & $41.27 \pm 0.17$ & $41.41 \pm 0.11$ & $41.47 \pm 0.10$ \\
\hline$R_{e}$ & $20.63 \pm 0.13$ & $20.86 \pm 0.16$ & $20.91 \pm 0.12$ & $20.90 \pm 0.10$ \\
\hline$R_{\mu}$ & $20.95 \pm 0.12$ & $20.64 \pm 0.11$ & $20.85 \pm 0.12$ & $20.796 \pm 0.073$ \\
\hline$R_{\tau}$ & $20.68 \pm 0.12$ & $20.64 \pm 0.16$ & $20.71 \pm 0.17$ & $21.00 \pm 0.11$ \\
\hline$A_{F B}^{o}(e)$ & $0.0218 \pm 0.0055$ & $0.0221 \pm 0.0073$ & $0.0125 \pm 0.0070$ & $0.0081 \pm 0.0051$ \\
\hline$A_{F B}^{o}(\mu)$ & $0.0192 \pm 0.0039$ & $0.0168 \pm 0.0030$ & $0.0164 \pm 0.0041$ & $0.0137 \pm 0.0027$ \\
\hline$A_{F B}^{o}(\tau)$ & $0.0217 \pm 0.0044$ & $0.0210 \pm 0.0057$ & $0.0305 \pm 0.0073$ & $0.0183 \pm 0.0035$ \\
\hline$A_{e}$ & $0.129 \pm 0.017$ & $0.136 \pm 0.027$ & $0.157 \pm 0.021$ & $0.134 \pm 0.016$ \\
\hline$A_{\tau}$ & $0.136 \pm 0.015$ & $0.148 \pm 0.022$ & $0.150 \pm 0.016$ & $0.134 \pm 0.013$ \\
\hline
\end{tabular}

Table I. The experimental data from the four LEP Collaborations. 


\begin{tabular}{lcccc}
$\alpha_{s}$ & 0.113 & 0.125 & 0.127 & 0.130 \\
$m_{h}(\mathrm{GeV})$ & 100 & 100 & 500 & 1000 \\
\hline \hline$\Gamma_{z}(\mathrm{MeV})$ & 2495.8 & 2502.3 & 2498.0 & 2496.5 \\
\hline$\sigma_{\text {had }}(\mathrm{nb})$ & 41.510 & 41.448 & 41.439 & 41.427 \\
\hline$R_{e}$ & 20.703 & 20.783 & 20.785 & 20.795 \\
\hline$R_{\mu}$ & 20.703 & 20.783 & 20.785 & 20.795 \\
\hline$R_{\tau}$ & 20.750 & 20.831 & 20.833 & 20.843 \\
\hline$A_{F B}^{o}(e)$ & 0.0174 & 0.0174 & 0.0158 & 0.0151 \\
\hline$A_{F B}^{o}(\mu)$ & 0.0174 & 0.0174 & 0.0158 & 0.0151 \\
\hline$A_{F B}^{o}(\tau)$ & 0.0174 & 0.0174 & 0.0158 & 0.0151 \\
\hline$A_{e}$ & 0.1524 & 0.1524 & 0.1452 & 0.1419 \\
\hline$A_{\tau}$ & 0.1524 & 0.1524 & 0.1452 & 0.1419 \\
\hline & & & &
\end{tabular}

Table II. We report the theoretical predictions at various values of $\alpha_{s}\left(M_{z}\right)$ and $m_{h}$ for a fixed top-quark mass $m_{t}=180 \mathrm{GeV}$. These predictions have been obtained with the computer code TOPAZ0 by G. Montagna, O. Nicrosini, G. Passarino, F. Piccinini and R. Pittau. 


\section{ALEPH}

\begin{tabular}{lcccc}
$\alpha_{s}$ & 0.113 & 0.125 & 0.127 & 0.130 \\
$m_{h}(\mathrm{GeV})$ & 100 & 100 & 500 & 1000 \\
\hline \hline$\Gamma_{z}$ & 0.25 & 2.46 & 0.68 & 0.32 \\
\hline$\sigma_{\text {had }}$ & 1.21 & 2.96 & 3.28 & 3.72 \\
\hline$R_{e}$ & 0.32 & 1.38 & 1.42 & 1.61 \\
\hline$R_{\mu}$ & 4.24 & 1.94 & 1.89 & 1.67 \\
\hline$R_{\tau}$ & 0.34 & 1.58 & 1.62 & 1.84 \\
\hline$A_{F B}^{o}(e)$ & 0.64 & 0.64 & 1.19 & 1.48 \\
\hline$A_{F B}^{o}(\mu)$ & 0.21 & 0.21 & 0.76 & 1.10 \\
\hline$A_{F B}^{o}(\tau)$ & 0.96 & 0.96 & 1.80 & 2.25 \\
\hline$A_{e}$ & 1.89 & 1.89 & 0.91 & 0.58 \\
\hline$A_{\tau}$ & 1.20 & 1.20 & 0.38 & 0.15 \\
\hline \hline TOTAL $\chi^{2}$ & 11.2 & 15.2 & 13.9 & 14.7 \\
\hline
\end{tabular}

Table III. Individual and total $\chi^{2}$ from the ALEPH Collaboration at various values of $\alpha_{s}\left(M_{z}\right)$ and $m_{h}$ for $m_{t}=180 \mathrm{GeV}$. 


\section{DELPHI}

\begin{tabular}{lcccc}
$\alpha_{s}$ & 0.113 & 0.125 & 0.127 & 0.130 \\
$m_{h}(\mathrm{GeV})$ & 100 & 100 & 500 & 1000 \\
\hline \hline$\Gamma_{z}$ & 0.10 & 2.22 & 0.50 & 0.19 \\
\hline$\sigma_{\text {had }}$ & 1.99 & 1.10 & 0.99 & 0.85 \\
\hline$R_{e}$ & 0.96 & 0.23 & 0.22 & 0.16 \\
\hline$R_{\mu}$ & 0.33 & 1.69 & 1.74 & 1.99 \\
\hline$R_{\tau}$ & 0.47 & 1.42 & 1.45 & 1.61 \\
\hline$A_{F B}^{o}(e)$ & 0.41 & 0.41 & 0.74 & 0.92 \\
\hline$A_{F B}^{o}(\mu)$ & 0.04 & 0.04 & 0.11 & 0.32 \\
\hline$A_{F B}^{o}(\tau)$ & 0.40 & 0.40 & 0.83 & 1.07 \\
\hline$A_{e}$ & 0.37 & 0.37 & 0.12 & 0.05 \\
\hline$A_{\tau}$ & 0.04 & 0.04 & 0.02 & 0.08 \\
\hline \hline TOTAL $\chi^{2}$ & 5.1 & 7.9 & 6.7 & 7.2 \\
\hline
\end{tabular}

Table IV. The same as in Table III for the DELPHI Collaboration. 


\section{L3}

\begin{tabular}{lcccc}
$\alpha_{s}$ & 0.113 & 0.125 & 0.127 & 0.130 \\
$m_{h}(\mathrm{GeV})$ & 100 & 100 & 500 & 1000 \\
\hline \hline$\Gamma_{z}$ & 2.39 & 0.10 & 1.28 & 2.00 \\
\hline$\sigma_{\text {had }}$ & 0.83 & 0.12 & 0.07 & 0.02 \\
\hline$R_{e}$ & 2.98 & 1.12 & 1.09 & 0.92 \\
\hline$R_{\mu}$ & 1.50 & 0.31 & 0.29 & 0.21 \\
\hline$R_{\tau}$ & 0.06 & 0.51 & 0.52 & 0.61 \\
\hline$A_{F B}^{o}(e)$ & 0.49 & 0.49 & 0.22 & 0.14 \\
\hline$A_{F B}^{o}(\mu)$ & 0.06 & 0.06 & 0.02 & 0.10 \\
\hline$A_{F B}^{o}(\tau)$ & 3.22 & 3.22 & 4.05 & 4.45 \\
\hline$A_{e}$ & 0.05 & 0.05 & 0.32 & 0.52 \\
\hline$A_{\tau}$ & 0.02 & 0.02 & 0.09 & 0.26 \\
\hline \hline TOTAL $\chi^{2}$ & 11.6 & 6.0 & 8.0 & 9.2 \\
\hline
\end{tabular}

Table V. The same as in Table III for the L3 Collaboration. 


\section{OPAL}

\begin{tabular}{lcccc}
$\alpha_{s}$ & 0.113 & 0.125 & 0.127 & 0.130 \\
$m_{h}(\mathrm{GeV})$ & 100 & 100 & 500 & 1000 \\
\hline \hline$\Gamma_{z}$ & 0.00 & 1.47 & 0.15 & 0.01 \\
\hline$\sigma_{\text {had }}$ & 0.16 & 0.05 & 0.10 & 0.18 \\
\hline$R_{e}$ & 3.88 & 1.37 & 1.32 & 1.10 \\
\hline$R_{\mu}$ & 1.62 & 0.03 & 0.02 & 0.00 \\
\hline$R_{\tau}$ & 5.17 & 2.36 & 2.30 & 2.04 \\
\hline$A_{F B}^{o}(e)$ & 3.32 & 3.32 & 2.28 & 1.88 \\
\hline$A_{F B}^{o}(\mu)$ & 1.88 & 1.88 & 0.60 & 0.27 \\
\hline$A_{F B}^{o}(\tau)$ & 0.07 & 0.07 & 0.51 & 0.84 \\
\hline$A_{e}$ & 1.32 & 1.32 & 0.49 & 0.24 \\
\hline$A_{\tau}$ & 2.00 & 2.00 & 0.74 & 0.37 \\
\hline \hline TOTAL $\chi^{2}$ & 19.4 & 13.9 & 8.5 & 6.9 \\
\hline & & & &
\end{tabular}

Table VI. The same as in Table III for the OPAL Collaboration. 
ALEPH + DELPHI + L3 + OPAL

\begin{tabular}{lcccc}
$\alpha_{s}$ & 0.113 & 0.125 & 0.127 & 0.130 \\
$m_{h}(\mathrm{GeV})$ & 100 & 100 & 500 & 1000 \\
\hline TOTAL $\chi^{2}$ & 47.3 & 43.0 & 37.1 & 38.1 \\
\hline
\end{tabular}

Table VII. Total $\chi^{2}$ for the four Collaborations.

\begin{tabular}{lcccc}
$\alpha_{s}$ & 0.113 & 0.125 & 0.127 & 0.130 \\
$m_{h}(\mathrm{GeV})$ & 100 & 100 & 500 & 1000 \\
\hline \hline ALEPH & 10.2 & 14.3 & 12.1 & 12.5 \\
\hline DELPHI & 4.7 & 7.5 & 5.9 & 6.2 \\
\hline L3 & 8.4 & 2.8 & 3.9 & 4.8 \\
\hline OPAL & 19.4 & 13.8 & 8.0 & 6.1 \\
\hline \hline TOTAL $\chi^{2}$ & 42.7 & 38.4 & 29.9 & 29.6 \\
\hline
\end{tabular}

Table VIII. Total $\chi^{2}$ for the four Collaborations by excluding the data for $A_{F B}^{o}(\tau)$. 
ALEPH+DELPHI+L3+OPAL

\begin{tabular}{ccccc}
$\alpha_{s}$ & 0.113 & 0.125 & 0.127 & 0.130 \\
$m_{h}(\mathrm{GeV})$ & 100 & 100 & 500 & 1000 \\
\hline \hline$m_{t}(\mathrm{GeV})=170$ & 46.3 & 38.4 & 38.3 & 41.2 \\
\hline$=180$ & 47.3 & 43.0 & 37.1 & 38.1 \\
\hline$=190$ & 51.8 & 50.4 & 38.9 & 37.5 \\
\hline
\end{tabular}

Table IX. Total $\chi^{2}$ for the four Collaborations at various values of $m_{t}$.

ALEPH+DELPHI+L3+OPAL

\begin{tabular}{ccccc}
$\alpha_{s}$ & 0.113 & 0.125 & 0.127 & 0.130 \\
$m_{h}(\mathrm{GeV})$ & 100 & 100 & 500 & 1000 \\
\hline \hline$m_{t}(\mathrm{GeV})=170$ & 40.7 & 32.8 & 29.7 & 31.2 \\
\hline$=180$ & 42.7 & 38.4 & 29.9 & 29.6 \\
\hline$=190$ & 50.1 & 46.6 & 32.9 & 30.3 \\
\hline
\end{tabular}

Table X. Total $\chi^{2}$ for the four Collaborations at various values of $m_{t}$ by excluding the data for $A_{F B}^{o}(\tau)$. 